\title{
Dyclonine Hydrochloride
}

National Cancer Institute

\section{Source}

National Cancer Institute. Dyclonine Hydrochloride. NCI Thesaurus. Code C66875.

The hydrochloride salt of Dyclonine, an unclassified compound with local anesthetic

effect. Dyclonine reversibly binds to activated sodium channels on the neuronal

membrane, thereby decreasing the neuronal membrane's permeability to sodium ions,

leading to an increased threshold for excitation. This reversibly stabilizes the membrane

and inhibits depolarization, leading to the failure of a propagated action potential and

subsequent conduction blockade. This results in a transient and reversible loss of

sensation in a localized area of the body. 